\title{
Diagnosing and treating rare lesions in a low resource setting: lessons from a hybrid epithelioid trophoblastic tumor and choriocarcinoma
}

\author{
Patrick K Akakpo ${ }^{1}$, Kofi Ulzen-Appiah ${ }^{1}$, Evans Agbeno ${ }^{2}$ and Leonard Derkyi-Kwarteng ${ }^{1}$ \\ Ghana Med J 2017; 51(4): 196-199 DOI: http://dx.doi.org/10.4314/gmj.v51i4.9 \\ ${ }^{1}$ Department of Pathology, School of Medical Sciences, University of Cape Coast, Cape Coast Teaching \\ Hospital, Cape Coast, Ghana ${ }^{2}$ Department of Obstetrics and Gynaecology, University of Cape Coast, Cape \\ Coast Teaching Hospital, Cape Coast
}

\author{
Corresponding author: Dr Patrick K. Akakpo \\ E-mail:k.p.akakpo@uccsms.edu.gh
}

Conflict of interest: None declared

\section{SUMMARY}

Objective: To raise awareness of the existence of a rare type of malignant trophoblastic tumor and discuss the diagnostic challenges and management of this lesion in a low resource setting.

Case report and intervention: A 35 -year -old $\mathrm{G}^{6} \mathrm{P}^{3}$ woman was referred to our facility on account of persistent vaginal bleeding due to a suspected incomplete miscarriage with a cervical mass. Her serum $\beta$-HCG was elevated $(36,900 \mathrm{mIU} / \mathrm{ml})$ and examination showed a bleeding cervical mass. An initial histopathological diagnosis of moderately differentiated squamous cell carcinoma was reviewed to epithelioid trophoblastic tumor resulting in an extra-fascial hysterectomy. A final histopathological diagnosis of hybrid Epithelioid Trophoblastic Tumor and Choriocarcinoma (ETT/CC) was made after external review and immunohistochemistry. She received subsequent chemotherapy.

Conclusion: Epithelioid trophoblastic tumor and its hybrids are difficult to diagnose. They may be diagnosed as moderately differentiated squamous cell carcinoma especially in low resource settings where cervical squamous cell carcinoma is relatively more common. A high index of suspicion, a serum $\beta$ HCG test and close collaboration between clinicians and pathologists can help make the diagnosis.

Funding: None

Keywords: Epithelioid trophoblastic tumour, choriocarcinoma, diagnostic challenges, low resource setting

\section{INTRODUCTION}

Gestational trophoblastic diseases (GTD) are characterized by abnormal proliferation of placental trophoblasts and include benign and malignant lesions. ${ }^{1}$ The malignant lesions are often referred to as Gestational Trophoblastic Tumours/Neoplasms (GTN). Epithelioid trophoblastic tumor is a rare neoplasm and represents the most recent addition to the gestational trophoblastic tumor category. ${ }^{2}$ ETT was first characterized in 1998 as a rare form of trophoblastic disease composed of intermediate trophoblasts, cells with features that made it distinct from placental-site trophoblastic tumor (PSTT) and choriocarcinoma (CC). ${ }^{3}$ ETT is easily confused histopathologically with squamous cell carcinoma because of its frequent involvement of the lower uterine segment or endocervix, its epithelioid histologic appearance, and expression of $\mathrm{p} 63$ and cytokeratins. ${ }^{3}$
Since its characterization as an entity, only up to one hundred (100) cases have been reported in the literature worldwide with 2 in South Africa. ${ }^{1,2,4}$ Hybrids of the entities that make up GTN exist, the commonest being a combination of ETT and CC and CC and PSTT., Though there are many reports suggesting that gestational trophoblastic GTDs including GTNs are more common in Africa, with the exception of the two (2) ETTs reported in South Africa, we are unaware of any other cases of ETT or its hybrids reported in SubSaharan Africa. ${ }^{7,-11}$ Suggestive clinical signs and symptoms such as bleeding per vaginam, elevated $\beta$ Human Chorionic Gonadotropin ( $\beta-\mathrm{HCG})$ and suggestive radiological findings combined with thorough histopathological examination are required in the diagnosis, management and follow up of patients with rare GTNs and ensure a favorable outcome. ${ }^{12}$ 


\section{Case Report}

\section{CASE REPORT}

A 35 -year -old Gravida 6, Para 3 (2 Alive, 1 Dead) woman presented to a peripheral hospital with an eight (8) week history of spontaneous vaginal bleeding with lower abdominal pain. She was referred to our facility because of persistent vaginal bleeding after three (3) manual vacuum evacuations for what was thought to be an incomplete miscarriage and a cervical mass thought to be a leiomyoma. There was no histopathological examination of the evacuation specimens. On arrival at our facility, she could not remember her last menstrual period and her past obstetric history was unremarkable with no history of gestational trophoblastic disease. Her first and second pregnancies resulted in planned vaginal deliveries at term with no complications. Her third pregnancy ended as a second trimester loss at six (6) months. Her fourth and fifth pregnancies were lost in the first trimester. Her most recent pregnancy event was a spontaneous abortion, three months prior to this presentation. Physical examination showed a stable but pale woman with a pelvic mass, about fourteen (14) weeks size, mildly tender and slightly mobile but one could not go below the mass. There was no obvious superficial pelvic lymphadenopathy on palpation. Vaginal speculum exam showed a hemorrhagic cervical mass that bled on contact. Urine pregnancy test was positive and initial serum $\beta$-HCG was elevated, 36,900 $\mathrm{mIU} / \mathrm{ml}(5-270,000 \mathrm{mIU} / \mathrm{ml})$. A pelvic ultrasound scan showed an empty uterus measuring 10.76x 6.02x $8.45 \mathrm{~cm}$ with a heterogeneous well-defined lesion in the cervical region measuring $6.34 \times 6.21 \times 6.94 \mathrm{~cm}$. The endometrium and adnexa were normal. A cervical curettage was done.

Histopathologically, the first cervical curettage specimen was signed out as suggestive of a moderately differentiated squamous cell carcinoma. A second curettage specimen was requested and submitted because the first sample had only few viable areas based on which the initial diagnosis of moderately differentiated squamous cell carcinoma was made. This was then reviewed together with the first biopsy and in the context of the elevated $\beta$ HCG, a diagnosis of Epithelioid Trophoblastic Tumor (ETT) was made. The patient then had an extra fascial total abdominal hysterectomy with conservation of both ovaries. The excised specimen showed a hemorrhagic $8 \times 6 \mathrm{~cm}$ mass confined to the cervix with histomorphology of ETT (Figure 1). The specimen was sampled per protocol and the hematoxylin and eosin stained sections reviewed with the initial biopsies. A diagnosis of ETT was made with a note to seek external consultation and carry out immunohistochemistry.
External consultation with immunohistochemistry (Figure 2) concluded this was a hybrid Epithelioid Trophoblastic Tumor and Choriocarcinoma (ETT/CC).

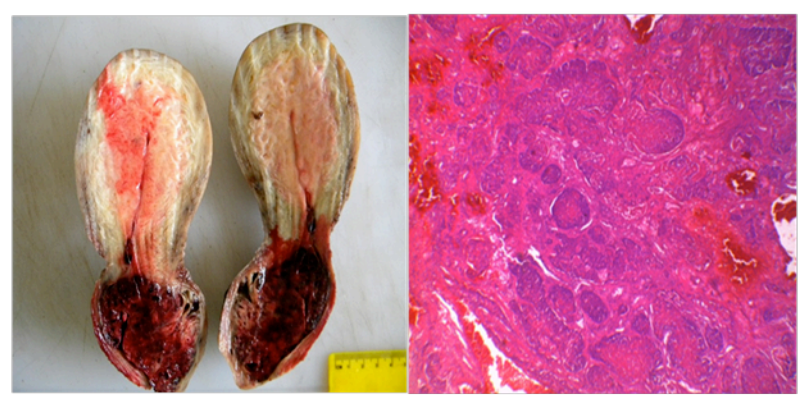

A

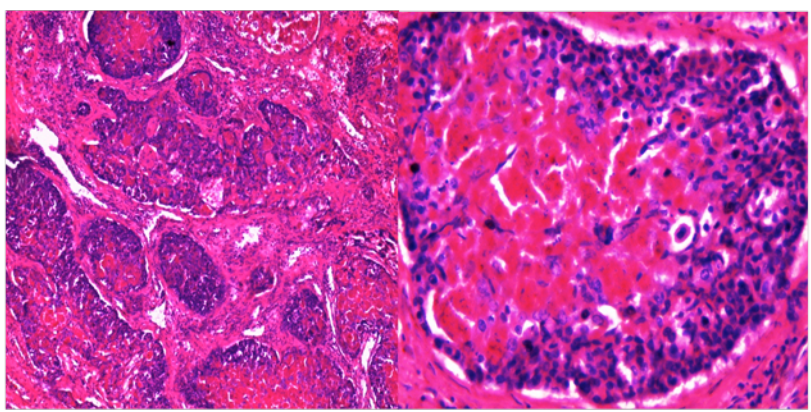

Figure 1 Showing gross and microscopic appearances of the tumor in the cervix. A (Gross location of tumor in the cervix) B, C, D (H\&E: epithelioid islands with hemorrhage)

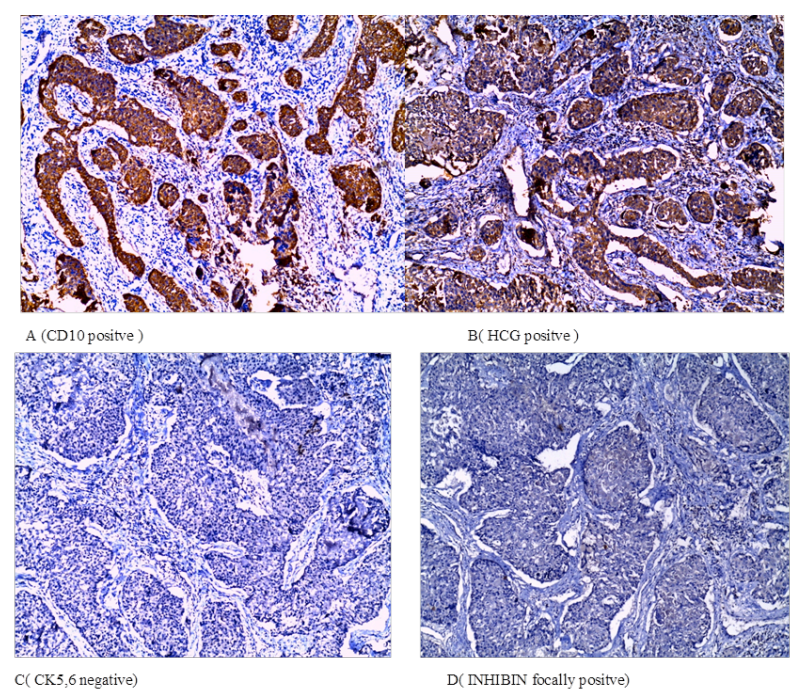

Figure 2 showing immunohistochemical panel.

Fifteen (15) days postoperatively, the patient's serum $\beta$ HCG dropped to $290.2 \mathrm{mIU} / \mathrm{ml}$ and then to $12 \mathrm{mIU} / \mathrm{ml}$ without chemotherapy. 


\section{Case Report}

After being lost to follow up for 4months without chemotherapy, the $\beta \mathrm{HCG}$ rose to $61.2 \mathrm{mIU} / \mathrm{ml}$ at which point chemotherapy with IV methotrexate was started resulting in a drop to $36.94 \mathrm{mIU} / \mathrm{ml}$ after a second cycle. There was however a rise in the $\beta$ HCG to $59.24 \mathrm{mIU} / \mathrm{ml}$ after the third cycle of chemotherapy requiring referral to the oncologist.

\section{DISCUSSION}

Our patient was in her reproductive years. Clinically, it is reported that ETT and hybrid ETTs primarily occur in reproductive-age women, though cases have been reported in postmenopausal women., ${ }^{3,5,6}$ ETT-like gestational $\mathrm{CC}$ occurs following a prior gestation with time from gestation to presentation reported to range from 1 year to as long as 18 years. ${ }^{3}$ The antecedent gestational events are reported to vary, including full term deliveries, spontaneous abortions and molar gestations with an average interval between the antecedent gestation and the development of the tumor reported as 6.2 years in one study. ${ }^{2}$ Hybrid GTNs similarly arise after pregnancy events and approximately one third of ETTs arise following a spontaneous abortion or hydatidiform mole, and majority after a full-term pregnancy., ${ }^{3,6}$ The index patient had three spontaneous abortions in the past following two previous term deliveries. The interval between the last spontaneous abortion, and her recent presentation to the hospital was 3 months. With no histopathological examination of previous curettage specimens, we are unable to classify the antecedent lesions histopathologically. It underscores the need to examine all such specimens in a histopathology laboratory. The most common presenting symptom of ETT and hybrid GTNs is reported as vaginal bleeding which was present in this patient. Together with a positive pregnancy test due to elevated serum $\beta$-human chorionic gonadotropin, this informed the initial diagnosis of spontaneous abortion at the peripheral facility. 'Low' elevations in serum $\beta$-human chorionic gonadotropin levels are reported for pure ETTs with levels generally not exceeding $2500 \mathrm{mIU} / \mathrm{mL}$, unlike choriocarcinoma. $^{3}$ Our patient's initial serum $\beta-\mathrm{HCG}$ was $36,900 \mathrm{mIU} / \mathrm{ml}$, higher than values reported for ETT though in keeping with the final diagnosis of hybrid ETT / Choriocarcinoma. ${ }^{3}$ Serum $\beta-\mathrm{HCG}$ is pivotal in the diagnosis of GTNs. In low resource settings, a simple serum $\beta$-HCG test in women bleeding per vaginam will alert clinicians to the presence of GTNs.

An ultrasound suggested a well-defined cervical lesion but did not seem to play a significant role in the subsequent diagnosis.
The cervical leiomyoma was however not further investigated to be sure the diagnosis was correct. Lack of proper attention to the "cervical leiomyoma" led to delayed diagnosis and could have led to more serious consequences. It has however been reported that ultrasound in combination with Doppler are useful tools for diagnosing invasive GTNs, assessing treatment response, and detecting local recurrence. MRI is invaluable to assess extra uterine disease spread and complications. Chest radiograph, brain MRI, and body CT are primarily used to rule out metastatic disease. ${ }^{12}$ The ultrasound findings and the initial diagnosis of ETT however informed the decision to do an extra-fascial total abdominal hysterectomy.

The initial diagnosis of moderately differentiated squamous cell carcinoma following curettage is in line with reports that the gross morphology (cervical location), histo-morphology (pattern of invasion, necrosis and keratinoid debris) and cytological (epithelioid appearance) features of ETT mimic squamous cell carcinoma. ${ }^{2,3}$ One of such studies reported that, histopathologically, the most challenging differential diagnosis is to separate ETT from an invasive squamous cell carcinoma, particularly when it arises in the cervix or lower uterine segment as in this patient. ${ }^{2}$ In this patient the diagnosis was reviewed to ETT after additional tissue was submitted and the serum $\beta$-HCG level was brought to the attention of the reporting pathologist. This further emphasizes the importance of close collaboration between the pathologist and gynecologist in the management of GTNs.

The initial drop in serum $\beta$-HCG following surgery and later resistance to routine chemotherapy with methotrexate are both in line with the predominant ETT composition of this patient's tumor. ETTs are treated conventionally with hysterectomy. The later rise in serum $\beta$-HCG following surgery and prior to initiation of chemotherapy with methotrexate are in keeping with the component of choriocarcinoma and underscore the need to properly diagnose hybrid lesions since conventional treatment options for pure forms of GTNs will not suffice for hybrid GTNs. The later rise in serum $\beta-H C G$ even after methotrexate therapy may be due the documented resistance of ETTs to conventional chemotherapy and the documented resistance of some high grade GTNs to methotrexate therapy. ${ }^{6}$

\section{CONCLUSION}

Epithelioid trophoblastic tumour and its hybrids are difficult to diagnose. They may be diagnosed as moderately differentiated squamous cell carcinoma especially in low resource settings where cervical 
squamous cell carcinoma is relatively more common. A high index of suspicion, a serum $\beta$ HCG test, relevant immunohistochemistry and close collaboration between clinicians and pathologists can help make the diagnosis.

\section{ETHICAL CLEARANCE}

In our institution, ethical clearance is not required to report clinical cases once all identifiers have been removed from the report. Verbal consent was however sought from the patient to publish this report.

\section{CONTRIBUTION}

Dr Evans Agbeno and Dr Ulzen-Appiah saw the patient, did the initial biopsies and performed the surgery. Leonard Derkyi-Kwarteng and Patrick Kafui Akakpo reported the initial biopsies and also reviewed them. They also examined and reported the excision specimen. Leonard Derkyi-Kwarteng and Patick Kafui Akakpo reviewed all the histopathology slides before and after external consultation. Leonard Derkyi-Kwarteng and Patrick Kafui Akakpo reviewed the literature and wrote the manuscript. All the authors reviewed the manuscript and agreed on its content.

\section{ACKNOWLEDGEMENT}

We are grateful to Dr Vinita Parkash of Bridgeport Hospital, Yale School of Medicine, USA, Members of The International Society of Gynaecological Pathologists and all the Gynaecological Pathology Fellows on her list serve who contributed their expertise and time to help us diagnose this lesion. We thank the histotechnicians in the department of pathology of Korle $\mathrm{Bu}$ Teaching Hospital for preparing the slides for this case.

\section{REFERENCES}

1. Scott EM, Smith AL, Desouki MM and Olawaiye AB. Epithelioid Trophoblastic Tumor: A Case Report and Review of the Literature. Case Rep in Obs and Gyn; 2012, Article ID; 862472, 5 pages doi:10.1155/2012/862472

2. Oluwole F, Parkash V, Carcangiu M-L and Pei Hui P. Epithelioid trophoblastic tumor: clinicopathological features with an emphasis on uterine cervical involvement. Mod Pathol. 2006 (19), 75-82

3. Allison KH, Love JE, Garcia RL, Epithelioid Trophoblastic Tumor; Review of a Rare Neoplasm of the Chorionic-Type Intermediate Trophoblasts Arch Pathol Lab Med. 2006 (130), 1875-1877

4. Elhassan MMA, Wu H-T, Van Wijk AL, Epithelioid trophoblastic tumour: a report of two cases. South Afr J Gynaecol Oncol 2015; 7(1):3436

5. Chen B-J, Cheng C-J and Chen W-Y. Transformation of a post-cesarean section placental site nodule into a coexisting epithelioid trophoblastic tumor and placental site trophoblastic tumor: a case report. Diagnostic Pathology 2013, $8: 85$

6. Shen D-H, Khoo U S, Ngan HYS, Ng T-Y, Chau M-T, Xue W-C, Cheung A.N.Y (2003) Coexisting Epithelioid Trophoblastic Tumor and Choriocarcinoma of the Uterus Following a Chemoresistant Hydatidiform Mole. Arch Pathol Lab Med: 2003, 127(7), 291-293

7. Yakasai I, Abubakar I, Eze Y. Gestational Trophoblastic Diseases in a Teaching Hospital in Northern, Nigeria. American Journal of BioScience. 3(1) 2015,7-10. doi: 10.11648/j.ajbio.20150301.12

8. Mbamara SU, Obiechina NJA, Eleje GU, Akabuike CJ, Umeononihu OS. Gestational Trophoblastic Disease in a Tertiary Hospital in Nnewi, Southeast Nigeria. Nigerian Med Journ. 2009 50(4) http://www.ajol.info/index.php/nmj/article/view/54 447

9. Moodley M, Tunkyi K and Moodley J. Gestational trophoblastic syndrome: An audit of 112 patients. A South African experience. IJGC. 2003; 13(2), 234239. DOI: $10.1046 / \mathrm{j} .1525-1438.2003 .13027 . x$

10. Di Cintio E, Parazzini F, Rosa C, Chatenoud L, Benzi G, The epidemiology of gestational trophoblastic disease. General \& Diagnostic Pathology. 1997, 143(2-3):103-108

11. Ellenson HL, Pirog EC. The Female Genital Tract, Gestational Trophoblastic Disease In Robbins and Cotran Pathologic Basis of Disease. Kumar, Abbas, Fausto and Aster, editors. Saunders Elsevier 2010.

12. Dhanda S, Ramani S and Thakur M. Gestational Trophoblastic Disease: A Multimodality Imaging Approach with Impact on Diagnosis and Management Radiology Research and Practice 2014 (ID 842751) available at: http://dx.doi.org/10.1155/2014/842751 